\title{
ON-CHIP CHARACTERIZATION OF FLUIDS USING MICRO SURFACE PLASMON RESONANCE SENSORS
}

\author{
Anna L. Pyayt, Kishore Sundara-Rajan, Gabriel I. Rowe and Matilda A. Enlund, \\ University of Washington, Seattle, WA 98195
}

\begin{abstract}
The design of a Micro Surface Plasmon Resonance Sensor is proposed. The possibility of implementing the modified Kretschmann-Raether scheme on a chip is investigated. A moving waveguide is used to change the angle of incidence of the beam of light. The main advantages of this sensor are potentially low cost, simplicity of design and very high parallelization. It is possible to have large number of such sensors on one chip, each of them with distinct activated sensing surfaces. The modeling of the sensor, design optimization procedures, and fabrication are also discussed. Computer simulations are used to validate the design.
\end{abstract}

Keywords: Surface plasmon resonance, lab on chip, micro sensors, MEMS.

\section{INTRODUCTION}

Molecular recognition and real time chemical reaction kinetics are powerful instruments used for investigation of the fundamental properties of bio-chemical systems. Surface Plasmon Resonance (SPR) has traditionally been a key technique used to study such systems. The ability of SPR to detect very small changes in the concentration of the component under study and its extremely high sensitivity make it an ideal observation technique for many scientific and commercial applications.

The conventional SPR systems require a sophisticated apparatus and a complicated setup, thus rendering it to be expensive and non-portable. Though there has been a continued effort to integrate the various components of the setup and miniaturize the device ${ }^{1,2}$, the efforts have been mostly concentrated in the macro scale and very little work has been done on realizing comprehensive SPR systems on a micro-scale. We propose to bridge this gap, and take the first step towards the realization of a truly comprehensive SPR based micro chemical analyzer.

The proposed device can be realized on a chip and consist of an array of SPR sensors. The gold surfaces of the different sensors will have different activations in order to be able to detect different chemical components in the studied media.

\section{THEORETICAL ASPECTS OF SPR}

Non-radiative surface plasmon waves (SPW) were first proposed as solution to Maxwell's equations by Sommerfeld in $1909^{3}$. Laboratory configurations for optical observation of SPW using the method of attenuated total reflection were demonstrated in 1968 by Otto ${ }^{4}$ and Kretschmann ${ }^{5}$. The potential of SPR for characterization of thin films and monitoring processes at metal interfaces was recognized in the late seventies. In 1983, the use of SPR for gas detection and biosensing was demonstrated and has since been receiving continuously growing attention from the scientific community. A great deal of work has been done in the exploitation of SPR for optical biosensing and several companies have commercialized it.

Surface plasmons are quasi-particles that are created at the interface of a metal and dielectric. The electro-magnetic wave associated with these quasi-particles is a solution of Maxwell's equations under the following conditions: 1) the dielectric constant of the dielectric medium should be real and positive, 2) dielectric constant of the metal should consist of both real and imaginary components, 3) the real part of the complex dielectric constant must be negative and its modulus must be larger than that of the imaginary part. The imaginary component of the dielectric constant determines the dissipation factor of the medium and hence its loss angle. To observe a sharp dip in the 
reflectance versus angle curve, a low loss medium is desirable. Hence a medium that has relatively low imaginary part of the dielectric constant is used.

The electro-magnetic wave associated with the plasmon must satisfy the boundary conditions of Maxwell's equations. Therefore, surface plasmons can only be excited by p-polarized light. Another condition, which must be taken into consideration, is that the angle of light incidence must be larger than the angle of total internal reflection. Further more, the thickness of the metal layer influences the sensitivity of the setup. There is a tradeoff between too high absorption of energy in thicker metal layers and too short plasmon lifetime in thinner layers. Typical optimum thickness of the metal layer is approximately several tens of nanometers.

There are several different metals, which can be used in SPR sensors. Silver-based sensors have the best sensitivity, but usually people prefer to use gold. The two main reasons of utilizing gold in SPR are: 1) its resistance to oxidation, 2) its capability to accommodate coating with variety of binding molecules ${ }^{6}$. This led to the decision to use thin gold film as the sensing surface of the sensor.

The choice of the light source is the second important step in SPR sensor fabrication. Kretschmann-Raether scheme assumes measurements of reflectance of monochromatic light depending of angle of incidence. Therefore the light source should be monochromatic. The visible and near-infra red parts of spectrum are convenient for the experiments from the optical point of view because of availability of different light sources and detectors ${ }^{6}$. On the other hand, wavelengths shorter than $500 \mathrm{~nm}$ can not be used, because absorption by gold becomes too large. Therefore in SPR sensors with sensing surfaces made of gold films, light sources with wavelength longer than 600 $\mathrm{nm}$ are desirable. In this design wavelength is chosen to be $825 \mathrm{~nm}$.

For the three-layer system (prism/metal/media under study) and for the fixed light source wavelength the reflectivity R may be given by Fresnel's equations:

$$
\begin{gathered}
r_{i k}^{p}=\frac{\tilde{n}_{k} \cos \theta_{i}-\tilde{n}_{i} \cos \theta_{k}}{\tilde{n}_{k} \cos \theta_{i}+\tilde{n}_{i} \cos \theta_{k}} \\
r_{i k}^{p}=\frac{\frac{k_{z i}}{\tilde{\varepsilon}_{i}}-\frac{k_{z k}}{\tilde{\varepsilon}_{k}}}{\frac{k_{z i}}{\tilde{\varepsilon}_{i}}+\frac{k_{z k}}{\tilde{\varepsilon}_{k}}} \\
r_{k i}^{p}=-r_{k i}^{p}
\end{gathered}
$$

Transmission:

$$
\begin{gathered}
t_{i k}^{p}=\frac{\tilde{n}_{i}}{\tilde{n}_{k}}\left(1+r_{i k}^{p}\right) \\
t_{k i}^{p}=\frac{\tilde{n}_{k}}{\tilde{n}_{i}}\left(1+r_{k i}^{p}\right)=\frac{\tilde{n}_{k}}{\tilde{n}_{i}}\left(1-r_{k i}^{p}\right) \\
t_{i k}^{p} t_{k i}^{p}=\left(1+r_{i k}^{p}\right)\left(1-r_{i k}^{p}\right)
\end{gathered}
$$

The total reflection of the three-layer model becomes

$$
\begin{gathered}
R=\left|r_{p r 12}^{p}\right|^{2}=\left|\frac{r_{p r 1}^{p}+r_{12}^{p} e^{2 i k_{z i} d_{1}}}{1+r_{p r 1}^{p} r_{12}^{p} e^{2 i k_{z i} d_{1}}}\right|^{2} \\
r_{i k}^{p}=\frac{\cos \theta_{i} / \tilde{n}_{i}-\cos \theta_{k} / \tilde{n}_{k}}{\cos \theta_{i} / \tilde{n}_{i}+\cos \theta_{k} / \tilde{n}_{k}} \\
n_{p r} \sin \theta_{p r}=\tilde{n}_{1} \sin \theta_{1}=\tilde{n}_{2} \sin \theta_{2} \\
\tilde{n}_{k} \cos \theta_{k}=\left(\tilde{n}_{k}^{2}-n_{p r}^{2} \sin ^{2} \theta_{p r}\right)^{1 / 2}
\end{gathered}
$$




$$
\begin{array}{r}
r_{i k}^{p}=\frac{\frac{\left(\tilde{\varepsilon}_{i}-n_{p r}^{2} \sin ^{2} \theta_{p r}\right)^{1 / 2}}{\tilde{\varepsilon}_{i}}-\frac{\left(\tilde{\varepsilon}_{k}-n_{p r}^{2} \sin ^{2} \theta_{p r}\right)^{1 / 2}}{\tilde{\varepsilon}_{k}}}{\frac{\left(\tilde{\varepsilon}_{i}-n_{p r}^{2} \sin ^{2} \theta_{p r}\right)^{1 / 2}}{\tilde{\varepsilon}_{i}}-\frac{\left(\tilde{\varepsilon}_{k}-n_{p r}^{2} \sin ^{2} \theta_{p r}\right)^{1 / 2}}{\tilde{\varepsilon}_{k}}} \\
r_{p r 1}^{p}=\frac{\cos \theta_{p r} / n_{p r}-\left(\tilde{\varepsilon}_{1}-n_{p r}^{2} \sin ^{2} \theta_{p r}\right)^{1 / 2} / \tilde{\varepsilon}_{1}}{\cos \theta_{p r} / n_{p r}+\left(\tilde{\varepsilon}_{1}-n_{p r}^{2} \sin ^{2} \theta_{p r}\right)^{1 / 2} / \tilde{\varepsilon}_{1}}
\end{array}
$$

Phase factor $k_{z 1} d_{1}=k_{1}\left(d_{1} \cos \theta_{1}\right)$ is optical path length

$$
k_{z 1} d_{1}=\frac{a}{c} d_{1}\left(\widetilde{\varepsilon}_{1}-n_{p r}^{2} \sin ^{2} \theta_{p r}\right)^{1 / 2}
$$

\section{SENSOR DESIGN}

To excite surface plasmons, the widely used and established Kretschmann-Raether scheme ${ }^{5,6}$ is used. The main modules of the sensor are: a) an array of alternating light sources and detectors, b) arrays of collimating lenses, c) moving waveguides, d) prisms, and e) micro-fluidic channels. In the proposed setup the whole sensor is realized on a single chip using MEMS techniques.

Figure 1 shows the schematic representation of the top view of the sensor. Light from an array of monochromatic light sources is coupled to moving input waveguides through collimating lenses. The oscillatory angular motion of the waveguide changes the incident angle of the beam of the light at the prism-metal interface. The light is redirected towards a collimating lens by a mirror after being reflected off the metal wall of the micro channel containing the fluid under study. The intensity of the collimated reflected beam is then sensed by an array of detectors. The variation of the intensity of the reflected beam with that of the angle of incidence divulges information regarding the chemical composition of the fluid in the micro-channel.

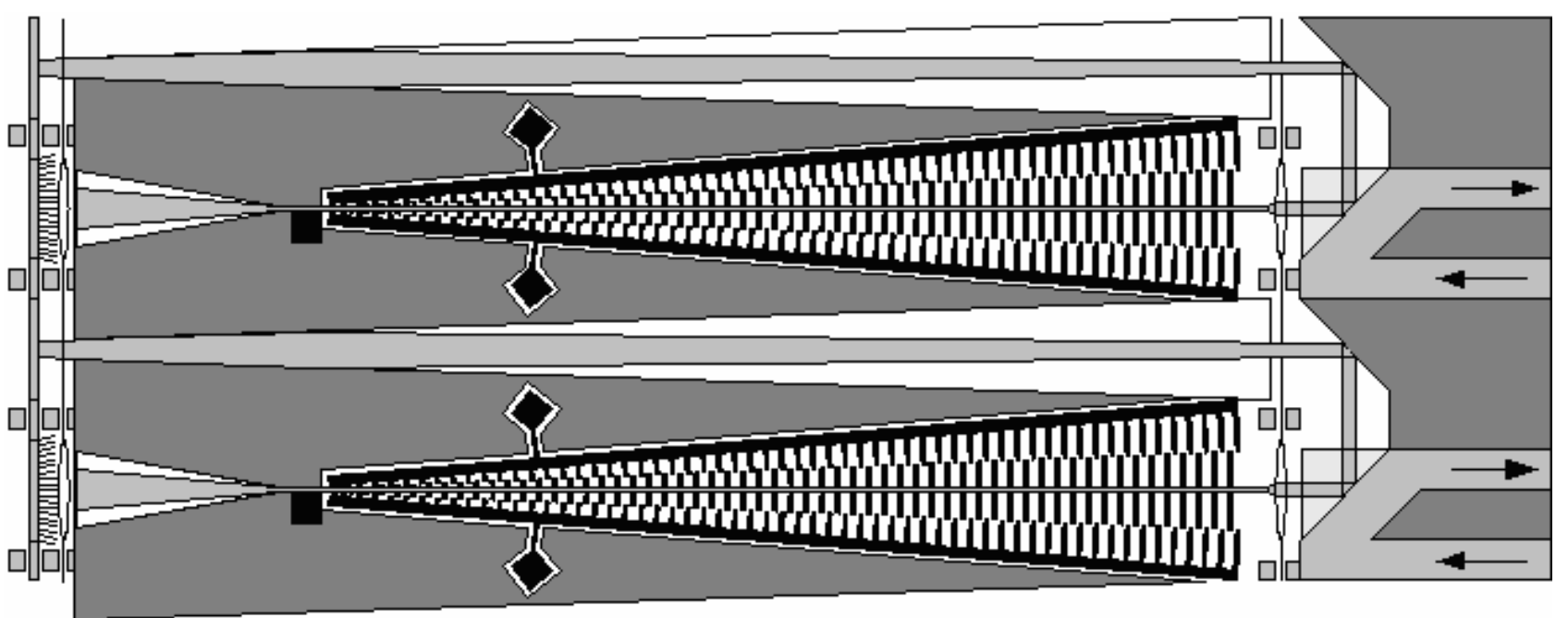

Figure 1. Schematic representation of the top view of the sensor.

The fabrication of the optical component arrays is a non-trivial process. These arrays are commercially available and are inexpensive ${ }^{7}$. The sensor fabrication process can be simplified by building part of the components of the sensor on a wafer using standard micro fabrication techniques, and then placing and bonding these readily available products into their prefabricated slots. 
To excite surface plasmons, a laser source with the wavelength of $825 \mathrm{~nm}$ is used. For a given wavelength of light, the chemical composition of the fluid in the channel determines the angle of incidence that is required to create conditions for plasmon resonance. At resonance, the reflected intensity of the parallel component of the incident light (p-polarization) is at its minimum. Hence monitoring the intensity variation of the reflected light at different angles of incidence, the optimal conditions for resonance can be determined.

The light from the on-chip sources exhibits high levels of divergence due to the small size of the source. An array of collimating lenses is placed on focal distance after the source to mitigate the effects of divergent light. The focal length of the lenses is $300 \mu \mathrm{m}$, diameter is $250 \mu \mathrm{m}$, and distance between two lenses in the array is $750 \mu \mathrm{m}$. Detectors are located on the same array as the light sources; the only difference between them is polarity. The moving part of the waveguide is $10 \mu \mathrm{m}$ high, $20 \mu \mathrm{m}$ wide, and $1000 \mu \mathrm{m}$ long. The prism is placed $300 \mu \mathrm{m}$ away from the lens. This prism has the shape of a triangle with $300 \mu \mathrm{m}$ side lengths. The walls of the channel are covered with a $50 \mathrm{~nm}$ gold film, which can be activated with different antibodies.

Figure 2 shows the variation of reflectance with change in the angle of incidence as described in (7). In this figure we can observe a dip at $42.77^{\circ}$. To obtain the best sensitivity of the sensor the value of the angle of incidence should be between these two points. Therefore we need to set the central angle of incidence equal to $42.77^{\circ}$ and the range of the motion up to $2.25^{\circ}$ in both directions.

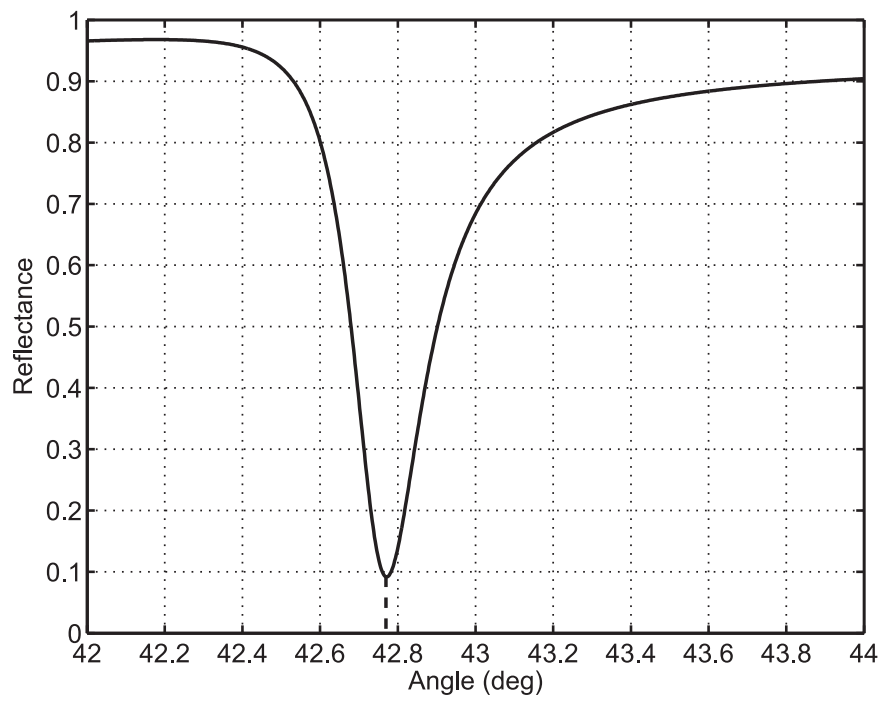

Figure 2. Variation of reflectance with change in angle of incidence of light with a wavelength of $825 \mathrm{~nm}$.

\section{FABRICATION}

The SPR sensor array can be manufactured using a combination of standard bulk and surface micromachining processes. The choice of the structural materials that can be used to manufacture the sensor are limited by constraints on their optical and physical properties as described in section 3. Silica not only meets the requirements, but also has established manufacturing processes, and hence is a logical choice of material. The technique to fabricate waveguides that deflect in the plane of the substrate were first published in ${ }^{8}$ and then refined in ${ }^{9}$. We intend to closely follow their technique with a few modifications. For waveguide fabrication we use three layers of silica with different levels of doping. The middle layer forms the core part of a waveguide and consists of higher doped silica than the cladding layers.

The first step is the deposition of a 3.8-micron thick layer of silica using plasma enhanced chemical vapor deposition. The waveguide region of this layer is then lightly doped with phosphorous. Following this, another layer of silica that is 2.8 micron thick is deposited. The waveguide region of this layer is then doped with high concentrations of phosphorous as shown in Figure 3(a). The waveguide is then patterned using $\mathrm{CHF}_{3} / \mathrm{O}_{2} \mathrm{RIE} \mathrm{etch}$. Height of the feature at the center of the core layer is $0.4 \mu \mathrm{m}$. The resulting structure is shown in Figure 3(b). Another 3.8-micron thick cladding layer of lightly doped silica is then deposited using PECVD as shown in Figure 
3(c). The three layers of silica are then anisotropically etched using RIE to define the waveguide and the actuator structures. The resulting waveguide structure is shown in Figure 3(d).

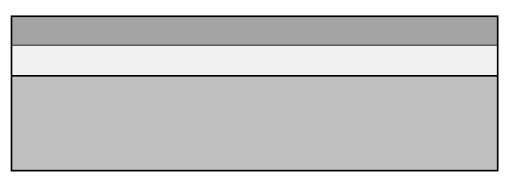

(A)

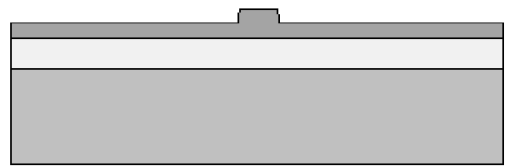

(B)

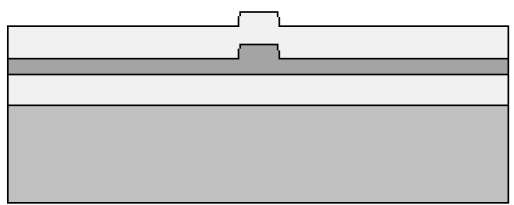

(C)

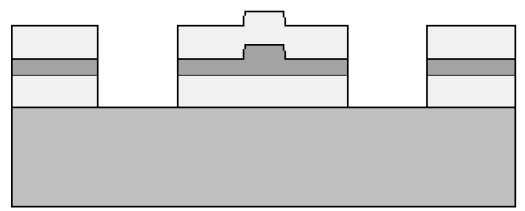

(D)

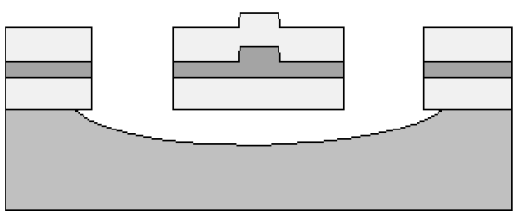

(E)

Figure 3. Fabrication of the waveguide.

While the structure formed using three different silica depositions is structurally strong enough for the actuator, it cannot be used to fabricate the prism. The intermittent doping processes require very high temperatures. When different layers of silica are treated to differential levels of heat treatment, their refractive indices vary. Hence, if a three-layer approach were used to fabricate the prism, each of the layers would have different refractive indices, and hence would introduce a large error in the output of the sensor. Hence, the prism and the channel are fabricated using separate silica deposition step. The prism and the channel can be patterned using RIE.

References ${ }^{9,8}$ suggest the use of lateral evaporation of gold to deposit metal on the sides of the fingers of the actuator. Therefore, to deposit gold on sides of fingers, mirror surface and walls of micro-channel gold evaporation can be used. Finally the structures are released using dry isotropic silicon etching. The released waveguide is shown in Figure 3(e). DRIE will be used for fabrication of the slots for the arrays of optical components.

\section{DESIGN PARAMETER OPTIMIZATION}

Computer simulations were used to determine the optimum parameters of the proposed system. Some of the design parameters are shown schematically in Figure 4. First of all, the limitations of the fabrication process were taken 
into consideration. Thicknesses of the cladding and core layers of the waveguide are determined by fact that singlemode waveguide is required for this sensor.

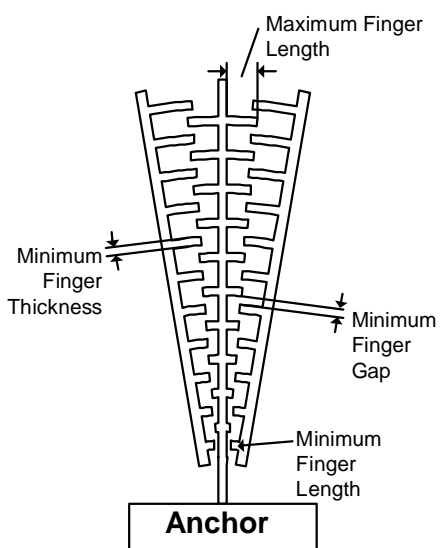

Figure 4. Schematic of the waveguide and the actuator detailing some of the design parameters.

Computations presented here assume that the electrostatic force on each finger is the same for all the fingers on the comb. The electrostatic force between the $\mathrm{n}^{\text {th }}$ and its neighboring finger in the moving part of the comb drive can be calculated as:

$$
\begin{gathered}
F_{e l n}=\frac{d W_{n}}{d x}=\frac{d W_{n}}{d \beta} \cdot \frac{d \beta}{d x} \\
W_{n}=\frac{1}{2} C_{n} V^{2}
\end{gathered}
$$

where,

$$
\begin{gathered}
C_{n}=\frac{\varepsilon_{0} \varepsilon_{r} A_{n}}{d} \\
A_{n} \approx h r_{n} \sin \beta \approx h r_{n} \beta \\
\Delta \beta=\frac{\Delta x}{r_{n}}
\end{gathered}
$$

hence,

$$
\begin{gathered}
\frac{d \beta}{d x}=\frac{1}{r_{n}} \\
F_{e l n}=\frac{d\left(\frac{1}{2} \frac{\varepsilon_{0} \varepsilon_{r} h r_{n} \beta}{d} V^{2}\right)}{d \beta} \cdot \frac{d \beta}{d x} \\
F_{e l n}=\frac{1}{2} \frac{\varepsilon_{0} \varepsilon_{r} h}{d} V^{2}
\end{gathered}
$$

Equation (21) shows that the force does not depend on the finger number, $n$. The resonant frequency, maximum angular displacement and the required force can be determined by modeling the system as a cantilever beam with distributed load. The resonant frequency $f_{b}$ of the beam is given by:

$$
f_{b}=\frac{1}{2 \pi} \sqrt{\frac{K_{s p r}}{m_{b}+m_{f}}}
$$


where $K_{s p r}$ is a spring constant of the system, $m_{b}=L w h \rho$ is the mass of the beam, and $m_{f}$ is the total mass of the fingers that are attached to the cantilever beam. The total mass of the fingers was calculated based on the number of fingers and their location.

It is assumed that all the fingers are uniformly distributed along the comb-drive. This assumption is necessary in order to maintain a constant force along the length of the comb. The number of fingers needed, $n$, is dependent on the applied voltage $V$, the generated force $F$ and the distance, $d$, between the fingers of the moving part of the combdrive and the anchored part of the com-drive.

$$
n=\frac{F_{e l} d}{\varepsilon_{0} \varepsilon_{r} h V^{2}}
$$

To calculate the spring constant of the system formula for the spring constant of a cantilever beam is used.

$$
K_{s p r}=\frac{2 E w^{3} h}{L^{3}}
$$

$F_{s p r}$, which is equal to the electrostatic force $F_{e l}$, is the spring force of a beam with the length $L$, width $w$ and height $h$. $E$ is the Young's Modulus of the material-in our case silica. $\theta$ is the angle of the maximum deflection of the beam.

Due to gravity, deflection of the beam is:

$$
F_{s p r}=k \cdot \Delta y=\frac{4 E w^{3} h \theta}{L^{2}}
$$

$$
d_{g}=\frac{\left(m_{b}+m_{f}\right) \cdot g}{K_{s p r}}
$$

This deflection can be neglected as it is approximately $0.2 \mu \mathrm{m}$ and is too small to cause considerable changes in light propagation.

In the design of an SPR device based on moveable waveguide structure, many constraints dictate the design of important features. For instance, the waveguide core and cladding indices of refraction set a critical angle defined in (27). We fabricate our sensor using silica-on-silicon technology, where the waveguide core has a refractive index $n_{\text {core }}=1.47$ and the cladding $-n_{\text {cladding }}=1.46$. This gives a critical angle, $\theta_{c}$, of $83.31^{\circ}$.

$$
\theta_{c}=\sin ^{-1}\left(\frac{n_{\text {cladding }}}{n_{\text {core }}}\right)=83.31^{\circ}
$$

The numerical aperture is determined by the following equation and describes the angle of acceptance and emission from the waveguide.

$$
N A=\sqrt{\mathrm{n}_{\text {core }}^{2}-\mathrm{n}_{\text {cladding }}^{2}}=0.171
$$

Once the desired angle of deflection at the end of the beam is calculated, the Euler-Bernoulli equation for the deflection of the cantilever beam under a distributed load, $p$, can be solved.

$$
\Theta(x)=-\frac{p x\left(3 L^{2}-3 x L+x^{2}\right)}{6 E I}
$$

where $\mathrm{x}$ is a distance from the anchored end of the beam, $\mathrm{L}$ - its length and $\mathrm{I}$ - bending momentum.

The maximum deflection is:

$$
\Theta_{\max }=\Theta(L)=-\frac{p L^{3}}{6 E I}
$$

For ease of use, it is desirable to minimize the operational voltage of the sensor. The voltage required to deliver the force needed is related to many important factors in the design and is shown in the equation below.

$$
F_{s}=F_{e l}=\frac{n \varepsilon_{0} \varepsilon_{r} h V^{2}}{d}
$$


The lower minimum for the length of the cantilever beam is determined by two constraints; the minimum acceptable bend radius of the cantilever to maintain low-loss transmission of the excitation light and the minimum feature size of an acceptable structural integrity for the comb fingers.

The relationship between the length of the beam, the excitation voltage and the finger width is given as:

$$
L\left(V, w_{f}\right)=\sqrt[3]{H(V) *\left(w_{f}+d\right)}
$$

where,

$$
H(V)=\frac{E w^{3} \tan (\theta) d}{\varepsilon_{0} \varepsilon_{r} V^{2}}
$$

Figure 5 shows the correlation between the length of the beam and voltage, as defined in (32), for different finger widths varying from $2 \mu \mathrm{m}$ in steps of $2 \mu \mathrm{m}$.

In this sensor design, $4 \mu \mathrm{m}$ wide fingers and $4 \mu \mathrm{m}$ finger gaps are used. For a cantilever beam of $1 \mathrm{~mm}, 62 \mathrm{fingers}$ fit on this length. The total displacement at the end of the beam is dictated by the maximum angle desired, 2.25 degrees. The longest finger is equal to 13 microns. The angle bounding the lengths of fingers, $\alpha$, is $1.69^{\circ}$. This means the finger lengths vary along the length of the cantilever as a function of this angle by $r \cdot \alpha$ (where $\alpha$ is in radians). The driving voltage required to reach the maximum displacement is $40 \mathrm{~V}$ and the resonant frequency is $28.75 \mathrm{kHz}$. The deflection due to gravity at rest is estimated at 0.07 microns by assuming a point load at the center of mass. The total mass of the released cantilever and comb structure is 0.45 micrograms.

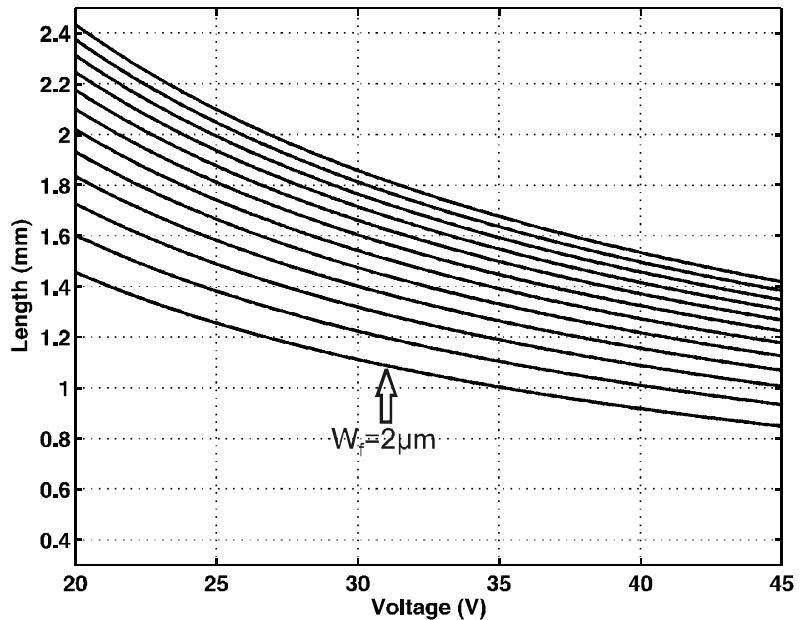

Figure 5. Relationship between required length and voltage for different finger width. The second line from the bottom is the 4 micron finger width, which was chosen for our characterization.

\section{CONCLUSION}

A viable design of a micro scale SPR sensor was proposed in this paper. Applications of this sensor include the ability to analyze chemical composition of samples flowing through microfluidic channels on the device. High parallelization and miniaturization are key advantages of this sensor to create an inexpensive, yet highly uniform design. This design appears to be powerful enough to analyze many samples yet at a fraction of the cost and size of larger SPR setups. While accuracy in larger setups may be greater, the high speed and small area under study make this device highly desirable. The speed and small size also seems very useful for consumers and military field operations. This paper is purely theoretical and requires experimental validation of the proposed design.

\section{ACKNOWLEDGEMENTS}

We would like to thank Prof. Karl Böhringer, University of Washington, Seattle, for his active participation and consultations, and furthermore for his courses on design and fabrication of micro-systems, which inspired this work. We would also like to thank Prof. Tim Chinowsky, and Prof. Lih Lin for their comments and advices. 


\section{REFERENCES}

1. Okamoto, T., Yamaguchi, I., and Kobayashi, T., "Local plasmon sensor with gold colloid monolayers deposited upon glass substrates", Optics Letters, 25, pp. 372-374, 3-15-2000.

2. Kao, F., Hong, X., and Takei, H., "Micro-surface plasmon resonance biosensing with cap-shaped gold nanoparticles", Focus on Microscopy 2003, Genova, Italy, 2003.

3. Hongo, L., "Surface Plasmon Resonance Biosensors: Development and Applications", 2000.

4. Otto, A., "Exitation of nonradiative surface plasma waves in silver by the method of frustrated total reflection.", Zeitschrift für Physik, 216, pp. 398-410, 1968.

5. Kretschmann, E and Raether, H., "Radiative decay of non-radiative surface plasmons excited by light", Zeitschrift für Naturforschung, 23A, pp. 2135-2136, 1968.

6. Homola, J., Yee, S. S., and Gauglitz, G., "Surface plasmon resonance sensors: review", Sensors and Actuators B-Chemical, 54, pp. 3-15, 1-25-1999.

7. "Monolithic Lenslet Modules", Adaptive Optics Associates, Inc., Ten Wilson Road, Cambridge, MA 02138, 2004.

8. Ollier, E., Labeye, P., and Revol, F., "Micro-Opto Mechanical Switch Integrated on Silicon", Electronics Letters, 31, pp. 2003-2005, 11-9-1995.

9. Ollier, E., "Optical MEMS devices based on moving waveguides", IEEE Journal of Selected Topics in Quantum Electronics, 8, pp. 155-162, 2002. 